\title{
Krajewski, C.; Wiegandt, C.-C. (Hrsg.) (2020): Land in Sicht. Ländliche Räume in Deutschland zwischen Prosperität und Peripherisierung
}

\author{
Larissa Deppisch (1)
}

Eingegangen: 19. April 2021 - Angenommen: 11. Mai 2021 - Online veröffentlicht: 3. Juni 2021

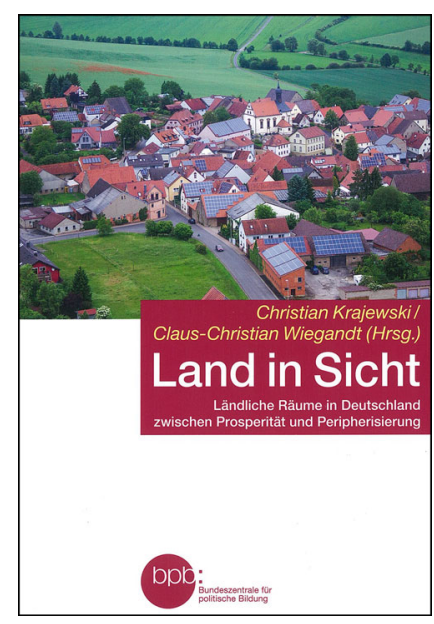

Ländliche Räume? Das sind doch diese dünn besiedelten, konservativen, verschlafenen Orte, wo es nur noch alte Leute gibt? Dort finden sich doch nur kleine landwirtschaftliche Betriebe, keine wichtigen Unternehmen und ein Laden schließt nach dem anderen. Aber schön ruhig ist es ja dort, die Landschaft ist ganz nett und die Leute halten noch zusammen! - An diesem stereotypen Bild arbeitet sich der 2020 erschienene Sammelband „Land in Sicht“ ab, der von den Geographen Christian Krajewski und Claus-Chris-

$\square$ Larissa Deppisch, Thünen-Institut für Ländliche Räume, Bundesallee 64, 38116 Braunschweig, Deutschland larissa.deppisch@thuenen.de

(c) (1) () 2021 Deppisch; licensee oekom verlag. This Open Access article is published under a Creative Commons Attribution 4.0 International License. tian Wiegandt bei der Bundeszentrale für politische Bildung herausgegeben wurde. In 26 Beiträgen aus der Geographie sowie der Soziologie, der Raumplanung und auch des Städtebaus wird die Thematik ländliche Räume in ihrer Komplexität häppchenartig behandelt.

Dabei nehmen die Beiträge immer wieder Bezug aufeinander, wie etwa auf die Typologien ländlicher Räume des Bundesinstituts für Bau-, Stadt- und Raumforschung (BBSR) sowie des Thünen-Instituts, die von Patrick Küpper und Antonia Milbert vorgestellt werden. Sie diskutieren, was unter ländlichen Räumen zu verstehen ist und warum sie stets ein Stadt-Land-Kontinuum statt eines Stadt-LandDualismus abbilden. Dem Stereotyp der sich ausschließlich entleerenden ländlichen Räume nehmen sich Stefan Kordel und Tobias Weidinger an. Sie zeigen auf, in welchen ländlichen Regionen Menschen zu- bzw. abwandern und welche Personengruppen aus welchen Motiven migrieren. So sehen die meisten Menschen, laut des Beitrags von Karl Martin Borns, ländliche Gemeinden als attraktive Wohnorte an. Ob Infrastrukturen vor Ort gut ausgebaut und zugänglich sind, ist hierfür von besonderer Relevanz.

In die aktuelle Versorgungssituation geben Christian Krajewski und Annett Steinführer einen Einblick und gehen zudem auf die verschiedenen Möglichkeiten der Verantwortungsübernahme - staatlich, privat oder ehrenamtlich für den Erhalt von Einrichtungen der Daseinsvorsorge ein. Über aktuelle Trends der Entwicklung ländlicher Infrastrukturen und darüber, welche Akteure bei der Transformation mitwirken, gibt Matthias Naumann Auskunft. Bernhard Faller und Jan Bendler betrachten die Gesundheitsversorgung genauer und zeigen die Diskrepanz zwischen tatsächlicher Verfügbarkeit bzw. Erreichbarkeit von Krankenhäusern sowie subjektiver Wahrnehmung und welche Gründe hinter dem Nachwuchsmangel in der Allgemeinmedizin stecken. 
Ähnlich wird auch im Beitrag von Holger Jahnke ein Lehrkräftemangel attestiert, für den zivilgesellschaftliche wie auch politische Lösungsstrategien vorgestellt werden. Eine potenzielle Antwort auf diverse Mängel der Daseinsvorsorgestruktur könnte im Hoffnungsträger Digitalisierung zu finden sein, so Michael Lobeck, der diskutiert, inwiefern durch diese alternative Versorgungsangebote geschaffen werden können. Auch Provinzstädte in ländlichen Räumen spielen für das Umland hinsichtlich der Sicherung der Versorgung, der Kommunikation und des Handels eine zentrale Rolle, wie Silke Weidner ausführt. Eine gute Infrastrukturausstattung verweist allerdings, so argumentieren Claudia Neu und Ljubica Nikolic, über die reine direkte Versorgungsfunktion hinaus. Gemeinsam mit einer kooperativen öffentlichen Verwaltung ist diese eine Grundvoraussetzung für die nachhaltige Schaffung sozialer Orte und damit auch für gesellschaftlichen Zusammenhalt.

Eine ebenfalls gesellschaftlich wichtige Versorgungsfunktion, die ländliche Räume einnehmen, ist die Produktion von Lebensmitteln. Peter Dannenberg verdeutlicht die agrarische Entwicklung sowie ihre Bedeutung für die Gesamtwirtschaft und wiegt Vor- und Nachteile konventioneller im Vergleich zu ökologischer Landwirtschaft ab. Ein weiterer wirtschaftlicher Bereich, der ländliche Räume prägt, ist der Tourismus. Marius Mayer, Manuel Woltering und Hubert Job benennen die relevantesten ländlichen Tourismusziele, wie hoch die regionale Tourismusintensität ausfällt und welche regionalökonomische Bedeutung der Tourismus für ländliche Räume hat. Doch mit Agrarbetrieben und Touristen lockenden Landschaften ist - entgegen des eingangs überspitzt gezeichneten Stereotyps - die wirtschaftliche Aktivität ländlicher Räume nicht ausgeschöpft. So hat ein Großteil der auf dem Weltmarkt handelnden mittelständischen deutschen Unternehmen ihren Sitz in ländlichen Räumen. Diese werden auch als Hidden Champions bezeichnet, wie Thilo Lang und Lukas Vonnahme erläutern. Sie zeigen die Motive von Unternehmen auf, in ländlichen Räumen zu verbleiben und welche Auswirkungen diese Entscheidungen für die lokale Situation haben. Entgegen der Vorstellung verschlafener Orte berichtet $\mathrm{Ga}$ briela Christmann vom Innovationspotenzial, welches ländlichen Räumen innewohnt sowie welche Voraussetzungen gegeben sein müssen, damit eine soziale Innovation gelingt und sich nachhaltig verstetigt.

Stets stellen die einzelnen Beiträge nicht nur aktuelle Entwicklungen der jeweiligen Aspekte ländlicher Räume vor, sondern gehen auch auf politische Folgen und Handlungsstrategien insbesondere vor dem Hintergrund des Anspruchs der Gleichwertigkeit der Lebensverhältnisse ein. Zudem zeigen Rainer Danielzyk und Ulrike Grabski-Kieron in ihren Beiträgen die Instrumente einer Politik ländlicher Räume auf nationaler wie auch europäischer Ebene. Damit schlägt der Band durchgehend eine Brücke zwischen wissenschaftlicher Analyse und politischer, raumplanerischer Praxis, die gesellschaftliche Prozesse rund um ländliche Räume ganzheitlicher betrachtet.

Das zentrale Fazit, welches die einzelnen Beiträge durchzieht und gleichzeitig übergreifend vermittelt wird, lautet: Den einen, vor allem negativ konnotierten, ländlichen Raum gibt es nicht. Stattdessen sind ländliche Räume heterogen. Diese Vielfalt ländlicher Räume wird zudem in verschiedenen Dimensionen deutlich: demographisch, infrastrukturell, wirtschaftlich und sozial. Ländliche Räume können also auch von Zuwanderung geprägt, strukturstark und wirtschaftlich aufstrebend sein. Der Untertitel des Bandes: „Ländliche Räume in Deutschland zwischen Prosperität und Peripherisierung“, vermittelt dies in aller Kürze bereits auf den ersten Blick.

Auch die Beiträge selbst sind stets kurzgehalten, was teilweise mit Vor-, aber auch Nachteilen hinsichtlich der Informationsvermittlung einhergeht. So sind die Beiträge auf die Darstellung von Forschungsergebnissen fokussiert. Damit geben die Autorinnen und Autoren eine schnell erfassbare Übersicht der aktuellen generellen Erkenntnisse ihres jeweiligen Themenfelds - vom zentralen Wissen der raumbezogenen (Sozial-)Wissenschaften sowie reflexiver raumplanerischer Fachdiskurse. Manchmal wird dabei allerdings die Erläuterung des methodischen Vorgehens, welches zu jenen Ergebnissen geführt hat, etwas vernachlässigt, wodurch die Analyse schwieriger nachvollziehbar ist. Das gewählte Format ermöglicht jedoch, in einem Buch die jeweiligen Quintessenzen diverser Themenfelder prägnant zusammenzutragen und dadurch der Komplexität ländlicher Räume gerecht zu werden. Gleichzeitig nehmen die Beiträge immer wieder Bezug aufeinander, sodass die einzelnen Themenfelder sowohl einzeln für sich erschlossen werden können, aber nicht zwangsläufig zusammenhangslos nebeneinanderstehen. Jene Querverbindungen regen eine Auseinandersetzung mit weiterführenden Themen an. Aus diesen Gründen eignet sich der Band vor allem als Überblickswerk und Einstiegsliteratur zum Thema ländliche Räume.

Vollständige bibliographische Angaben des rezensierten Werkes: Krajewski, C.; Wiegandt, C.-C. (Hrsg.) (2020): Land in Sicht. Ländliche Räume in Deutschland zwischen Prosperität und Peripherisierung. Bonn: Bundeszentrale für politische Bildung, Band 10362. 410 Seiten. Illustrationen. Diagramme. Karten. 